\title{
Tetrandrine Inhibits the Wnt/ $\beta$-Catenin Signalling Pathway and Alleviates Osteoarthritis: An In Vitro and In Vivo Study
}

\author{
Xindie Zhou, Weijun Li, Lifeng Jiang, Jiapeng Bao, Lijiang Tao, Jin Li, and Lidong Wu \\ Department of Orthopedics Surgery, The Second Affiliated Hospital of Medical College, Zhejiang University, Hangzhou 310000, China \\ Correspondence should be addressed to Lidong Wu; ldwu@yahoo.com
}

Received 27 November 2012; Accepted 3 February 2013

Academic Editor: Mei Tian

Copyright ( 2013 Xindie Zhou et al. This is an open access article distributed under the Creative Commons Attribution License, which permits unrestricted use, distribution, and reproduction in any medium, provided the original work is properly cited.

\begin{abstract}
There is currently no effective drug treatment for the early phase of osteoarthritis (OA), one of the most common senile diseases. The goal of this study was to investigate the protective effect of the tetrandrine (Tet) on OA, in vitro and in vivo. In an in vitro experiment, quantitative real-time polymerase chain reaction (qRT-PCR) was used to investigate changes in gene expression upon the addition of Tet in chondrocytes processed with IL-1 $\beta$; changes in protein profiles were assessed by Western blotting. In vivo, to determine whether Tet has the protective effects on articular cartilage, a rabbit anterior cruciate ligament transaction model of OA was established. Expression of matrix metalloproteinase and $\beta$-catenin genes increased significantly, while that of tissue inhibitor of metalloproteinase-1 decreased significantly in the OA group both in vivo and in chondrocytes. However, the changes of expression were reversed by Tet, and there was less cartilage degradation in vivo compared with the OA group, as assessed by histological and macroscopic observations. Thus, Tet may play a useful role in the treatment of OA through the Wnt/ $\beta$-catenin signalling pathway and has potential for the treatment of OA.
\end{abstract}

\section{Introduction}

Osteoarthritis (OA) is the most common joint disorder and is a major cause of pain, disability, and loss of quality of life. It is regarded as a degenerative disease and is associated with joint marginal osteophyte formation. It is believed that obesity, bone mass, joint injury and instability, developmental diseases, trauma, joint deformity, and age are common factors in OA, especially of the hip and knee joints [1]. Although $\mathrm{OA}$ is regarded primarily as a noninflammatory arthropathy, symptoms of local inflammation, as well as synovitis, are present in many patients and animal models of OA during cartilage destruction [2]. Interleukin-1 (IL1), originally known as a lymphokine, was first described in the context of cellular interactions in articular tissues as a monocyte/macrophage product that induced collagenase and prostaglandin production in synovial fibroblast cultures [3]. A previous study showed that IL-1 mediated marked downregulation of the matrix metalloproteinases (MMPs) in chondrocytes [4].

It is now generally accepted that chondrocyte senescence and destruction are the final results of abnormal biomechanical factors, and that biochemical and genetic factors also play important roles in the normal functional activities of these cells. MMPs have received much attention because they specifically degrade native collagens and proteoglycans. Levels of MMPs are increased in the cartilage of OA patients, and there is a decrease in levels of tissue inhibitor of metalloproteinase-1 (TIMP-1) [5]. Among these enzymes, MMP-1 and MMP-13 play major roles in degrading the components of the cartilage matrix, especially the aggrecans and collagens. Active stromelysin (MMP-3) also serves as an activator of latent collagenases and digests proteoglycan aggregates in human articular cartilage [6]. TIMPs act as inhibitors of MMPs. TIMP levels are elevated in OA cartilage, possibly reflecting an endogenous adaptive response to the increased levels of active proteinase activity [7].

Nonsteroidal anti-inflammatory drugs (NSAIDs), corticosteroids, and hyaluronan have been clinically used for the treatment of OA in the clinic. However, they fail to reverse cartilage damage, and a proportion of patients still progress and ultimately require surgery. Thus, there is a continuing need for better agents with which to treat OA [8].

Here, we investigated the activity of tetrandrine (Tet) in the protection of articular cartilage. Tet (International 
Union of Pure and Applied Chemistry name: 6,6 $6^{\prime}, 7,12-$ tetramethoxy-2,2' -dimethyl-1 $\beta$-berbaman; Chemical Abstracts Service number 518-34-3; $\mathrm{C}_{38} \mathrm{H}_{42} \mathrm{~N}_{2} \mathrm{O}_{6}$; molecular weight, 622.74988) is a bisbenzylisoquinoline alkaloid, purified from the root of Stephania tetrandrine of the Menispermaceae family. It has been used as an antihypertensive and antiarrhythmic agent as well as an operative agent in cardiovascular disease because of its calcium channelblocking effects, and it has been shown to exhibit antifibrotic activity in silicosis $[9,10]$. Early findings showed that Tet may have value in the therapy of chronic inflammatory diseases in which IL-1 or TNF play a role in pathogenesis $[11,12]$. Recent studies have shown that $\mathrm{Wnt} / \beta$-catenin signalling also participates in the response to mechanical injury to cartilage and that Tet may have an effect on OA through Wnt/ $\beta$-catenin signalling from a study of the effect of Tet on tumour growth in human colorectal cancer $[13,14]$. However, little is known about its possible use in the treatment of OA. In this study, we evaluated the effects of Tet on cartilage degradation following intra-articular injection in an experimental OA model and at $20 \mathrm{mg} / \mathrm{L}$ in an articular chondrocyte experiment; at this concentration, the effectiveness of Tet in vitro has been confirmed.

\section{Materials and Methods}

2.1. Reagents. Tet (Sigma, St. Louis, MO, USA) was dissolved in $0.01 \mathrm{~mol} / \mathrm{L} \mathrm{HCl}(1 \mathrm{mg}$ Tet dissolved in $10 \mathrm{uL} 0.01 \mathrm{~mol} / \mathrm{L}$ $\mathrm{HCl}$ to mother liquor) and diluted to $20 \mathrm{mg} / \mathrm{L}$ by Dulbecco's modified Eagle's medium (DMEM) for the in vivo test and by phosphate-buffered saline (PBS) for the in vitro test.

2.2. MTT Assays. Cells were cultured in a 96-well plate (8000/well). After incubation with various concentrations (5$100 \mathrm{mg} / \mathrm{L}$ ) of Tet for $24 \mathrm{~h}$ in a serum-free medium, MTT $(5 \mathrm{mg} / \mathrm{mL})$ was added $(20 \mathrm{uL} /$ well $)$. Cells were then incubated with MTT for $4 \mathrm{~h}$, and culture medium was removed and DMSO was added $(150 \mathrm{~mL} /$ well). Absorbance was measured at $570 \mathrm{~nm}$. This step was repeated for three times to get average results to reduce errors.

2.3. Primary Cell Culture and Treatment. The study was approved by the Institutional Animal Care and Use Committee of Zhejiang University (Hangzhou, China). Fourweek-old New Zealand white rabbits were sacrificed by air embolism. Immediately, cartilage harvested from the knee joints of rabbits under sterile conditions was digested with $0.25 \%$ pancreatic enzymes for $30 \mathrm{~min}$ to remove other tissues and cells, then digested with $0.2 \%$ collagenase II at $37^{\circ} \mathrm{C}$ for $4 \mathrm{~h}$, as described previously with minor modifications. Cells were grown to confluence in DMEM supplemented with $10 \%$ fetal bovine serum (FBS), $100 \mathrm{U} / \mathrm{mL}$ penicillin, and $100 \mathrm{mg} / \mathrm{mL}$ streptomycin at $37^{\circ} \mathrm{C}$ with $5 \% \mathrm{CO}_{2}$. Cells from the third passage were used. Experiments were performed after subconfluent cells were serum-starved overnight. Cells were seeded in six-well plates $\left(1 \times 10^{5} /\right.$ well $)$, and subconfluent cells were preincubated with three concentrations of Tet (derived from preliminary tests) for $1 \mathrm{~h}$ followed by stimulation with
IL-1 $\beta(10 \mathrm{ng} / \mathrm{mL})$ for $24 \mathrm{~h}$. Cells were then harvested and subjected to determination of MMP-1, MMP-3, MMP-13, and TIMP-1 mRNA expression levels to assess the optimum Tet concentration, which was then used in subsequent experiments.

2.4. Gene Expression Analysis. Total RNA was extracted from chondrocytes treated with various Tet concentrations and cartilage harvested from the in vitro test using the TRIzol reagent (Invitrogen, Carlsbad, CA, USA) according to the manufacturer's protocol. Total RNA $(600 \mu \mathrm{g}), 1 \mu \mathrm{L}$ of primer mix, and $1-\mu \mathrm{L}$ dNTPs $(10 \mathrm{mM})$ were added to a $200-\mu \mathrm{L}$ RNase-free centrifuge tube. DEPC-treated water was added $(15 \mu \mathrm{L})$, and the centrifuge tube was incubated on ice. The tube was then incubated at $70^{\circ} \mathrm{C}$ for $5 \mathrm{~min}$. Next, $4 \mu \mathrm{L}$ of $5 \times$ first-strand buffer, $2 \mu \mathrm{L}$ of $0.1 \mathrm{M}$ DTT, 25 units of RNase inhibitor, and 200 units of Superscript II reverse transcriptase (Invitrogen) were added. RNA was reverse-transcribed into cDNA. MMP-1, MMP-3, MMP-13, and TIMP-1 expression levels were quantified by quantitative real-time polymerase chain reaction (qRT-PCR) using the iCycler system (BioRad, Hercules, CA, USA) and iQ SYBR Green Supermix PCR kit (Bio-Rad), based on sequence information (Table 1). A parallel amplification with rabbit $18 \mathrm{~S}$ primers was carried out to normalise the expression data of the targeted gene transcripts. The relative levels of targeted gene expressions were calculated following the formula:

$$
2^{-(\Delta c t \text { target gene }-\Delta c t 18 \mathrm{~s} \text { rRNA })}
$$

2.5. Western Blot Analysis. Cells were rinsed with iced PBS, and total protein was extracted from three samples using a total protein extraction kit, then quantified with a BCA quantification kit. Proteins were resolved by sodium dodecyl sulphate polyacrylamide gel electrophoresis and then transferred onto polyvinylidene fluoride membranes. After blocking for $1 \mathrm{~h}$ with $5 \%$ milk in Tris-buffered salineTween, membranes were incubated with antibodies against MMP-3 (anti-MMP-3 antibody; Santa Cruz sc-6839, USA), TIMP-1 (anti-TIMP1 antibody; Abcam ab126847, USA), $\beta$ catenin, (anti-beta catenin antibody; Abcam ab83295, USA), and $\beta$-actin (Santa Cruz Biotechnology) overnight at $4^{\circ} \mathrm{C}$. Membranes were incubated with goat anti-mouse IgG-HRP and goat anti-rabbit secondary antibody at room temperature for $1 \mathrm{~h}$, and signals were detected using an Enhanced Chemiluminescence kit (GE Healthcare, Shanghai, China) with exposure to X-ray film (Kodak, Hangzhou, China).

2.6. Induction of $O A$ in Rabbits. Fifteen New Zealand white rabbits weighing $2.0 \mathrm{~kg}$ were used (Animal Centre of Zhejiang University). All experiments were conducted with the approval of Zhejiang University Animal Care and Use Committee.

Ten of the 15 rabbits underwent bilateral anterior cruciate ligament transections (ACLTs) on the knee joints to induce OA and were divided into two groups randomly. The other five rabbits (control group) received sham operations, which 
TABLE 1: Primers of targeted genes.

\begin{tabular}{|c|c|c|c|c|}
\hline Gene & Genbank accession & Primer sequences $\left(5^{\prime}\right.$ to $\left.3^{\prime}\right)$ & Size (bp) & Annealing $\left({ }^{\circ} \mathrm{C}\right)$ \\
\hline \multirow{2}{*}{ Rabbit MMP-1 } & \multirow{2}{*}{ M25663 } & F: CAGGAGCCTTCCCAAGAGGAA & \multirow{2}{*}{79} & \multirow{2}{*}{62} \\
\hline & & R: CTTGTCTCTTGCATATCAGGATGATG & & \\
\hline \multirow{2}{*}{ Rabbit MMP-3 } & \multirow{2}{*}{ NM_001082280 } & F: ACACCGGATCTGCCAAGAGA & \multirow{2}{*}{89} & \multirow{2}{*}{63} \\
\hline & & R: CTGGAGAACGTGAGTGGAGTCA & & \\
\hline \multirow{2}{*}{ Rabbit MMP-13 } & \multirow{2}{*}{ NM_001082037 } & F: CAGATGGGCATATCCСTCTAAGAA & \multirow{2}{*}{88} & \multirow{2}{*}{63} \\
\hline & & R: CCATGACCAAATCTACAGTCCTCAC & & \\
\hline \multirow{2}{*}{ Rabbit TIMP-1 } & \multirow{2}{*}{ AY829730 } & F: CAACTGCGGAACGGGCTCTTG & \multirow{2}{*}{102} & \multirow{2}{*}{63} \\
\hline & & R: CGGCAGCGTAGGTCTTGGTGAA & & \\
\hline \multirow{2}{*}{ Rabbit $\beta$-catenin } & \multirow{2}{*}{ DQ786777 } & F: CGTACGCACCATGCAGAACACAAA & \multirow{2}{*}{154} & \multirow{2}{*}{63} \\
\hline & & R: ATCCACTGGTGAACCGAGCATCTT & & \\
\hline \multirow{2}{*}{ Rabbit $18 S$} & \multirow{2}{*}{ EU236696 } & F: GACGGACCAGAGCGAAAGC & \multirow{2}{*}{119} & \multirow{2}{*}{62} \\
\hline & & R: CGCCAGTCGGCATCGTTTATG & & \\
\hline
\end{tabular}

involved opening the articular cavity and resuturing it without cutting the short anterior cruciate ligament. After surgery, all animals were returned to their cages; the limbs were not immobilised. At 1 month after surgery, the Tet group was given intra-articular injections of $0.3 \mathrm{~mL}$ Tet $(20 \mathrm{mg} / \mathrm{L})$ in both knees once per week for 6 weeks. The OA group was injected with $0.3 \mathrm{~mL}$ solvent alone in both knees under the same conditions (solvent: $10 \mu \mathrm{L}$ of $0.01 \mathrm{~mol} / \mathrm{L} \mathrm{HCl}$ dissolved in $50 \mathrm{~mL}$ of PBS). In the sham-operation group, no other procedures were conducted. Rabbits were sacrificed 7 days after the last injection.

2.7. Histological Examination. Five samples from each group were fixed in $4 \%$ paraformaldehyde [15], decalcified with $10 \%$ formic acid, buffered at $\mathrm{pH} 7.4$, dehydrated through a series of ethanol solutions, embedded in paraffin, cut into $3-\mu \mathrm{m}$ sections, and stained with safranin $\mathrm{O}$-fast green. The samples were scored for the degree of histological change using the Mankin score system [16]. Two independent researchers assessed the extent of histological cartilage damage in a blinded manner.

2.8. Statistical Analysis. All data were expressed as mean \pm standard deviation (SD). Statistical analysis of MTT assay data was performed by an unpaired $t$-test while histological and gene expression data were analyzed by a paired $t$-test. Differences were considered significant at $P<0.05$.

\section{Results}

3.1. Effects of Tet on Viability. The chondrocyte toxicities of $100,50,20,10$, and $5 \mathrm{mg} / \mathrm{L}$ Tet were assessed by MTT assay. Statistical analysis of MTT assay data was conducted using an unpaired $t$-test (Figure 1). Concentrations of $>20 \mathrm{mg} / \mathrm{L}$ had toxic effects on chondrocytes. As a result, $20 \mathrm{mg} / \mathrm{L}$ was selected as the optimum concentration and was used in subsequent experiments.

3.2. Effects of Tet on the Expression of MMP-1, MMP-3, $M M P-13$, TIMP-1, and $\beta$-catenin in Rabbit Chondrocytes

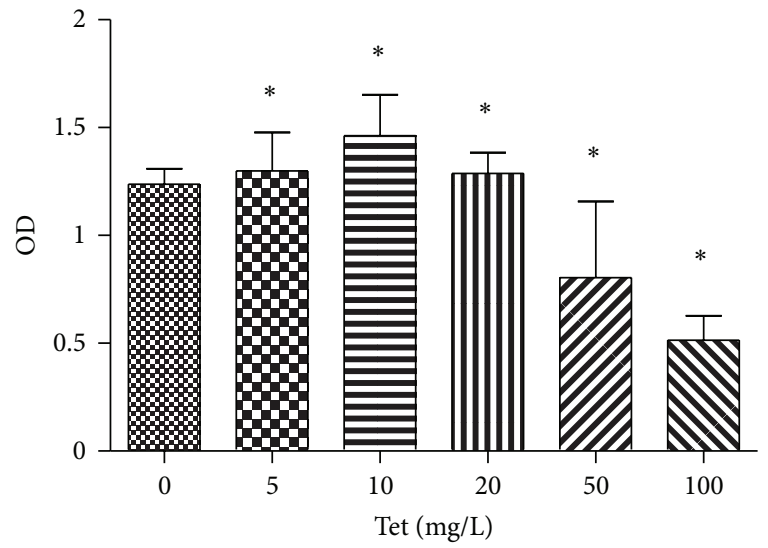

FIGURE 1: The effects of Tetrandrine (Tet) on chondrocyte proliferation as determined by the MTT assay. Chondrocytes $(8,000)$ were initially plated in each well of a 96-well plate and treated with Tet at $0,5,10,20,50,100 \mathrm{mg} / \mathrm{L}$. At $24 \mathrm{~h}$, the number of viable cells was measured by MTT assay. Values are mean \pm SD of three independent experiments. ${ }^{*}$ Statistical significance in viable cell numbers $(P<$ $0.05)$.

and Cartilage. qRT-PCR was performed to determine the expression levels of MMPs in chondrocytes. To investigate the effects of Tet on gene expression, chondrocytes were preincubated with Tet for $1 \mathrm{~h}$ prior to stimulation with IL$1 \beta$ for $24 \mathrm{~h}$. Chondrocytes stimulated with IL- $1 \beta$ showed induction of MMP-1, MMP-3, and MMP-13 gene expression, but downregulation of TIMP-1 expression (Figure 2(a)). Tet inhibited the IL- $1 \beta$-mediated induction of MMP-1, MMP-3, and MMP-13 gene expression and induced the expression of TIMP-1. We next examined the effects of IL- $1 \beta$ and Tet on protein expression of MMP-1, MMP-3, MMP-13, and TIMP1 in chondrocytes. The optimum Tet concentration was used (Figures 2(b) and 2(c)). Treatment with IL-1 $\beta$ resulted in the upregulation of MMP-1, MMP-3, and MMP-13 and the downregulation of TIMP-1 at the protein level. These effects were blocked by Tet. We identified similar changes in the analysis of the expression of the MMP, TIMP-1, and $\beta$-catenin genes in vivo (Figure 3(a)). 

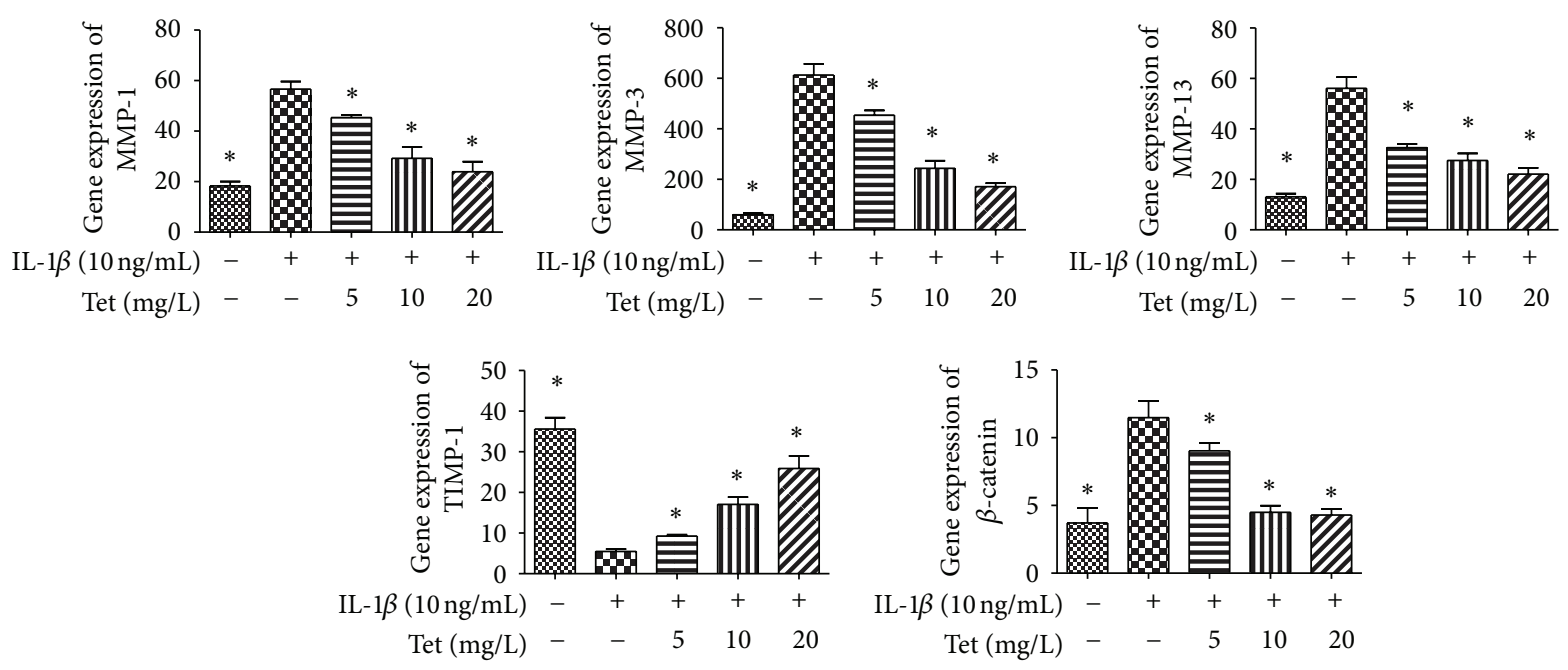

(a)
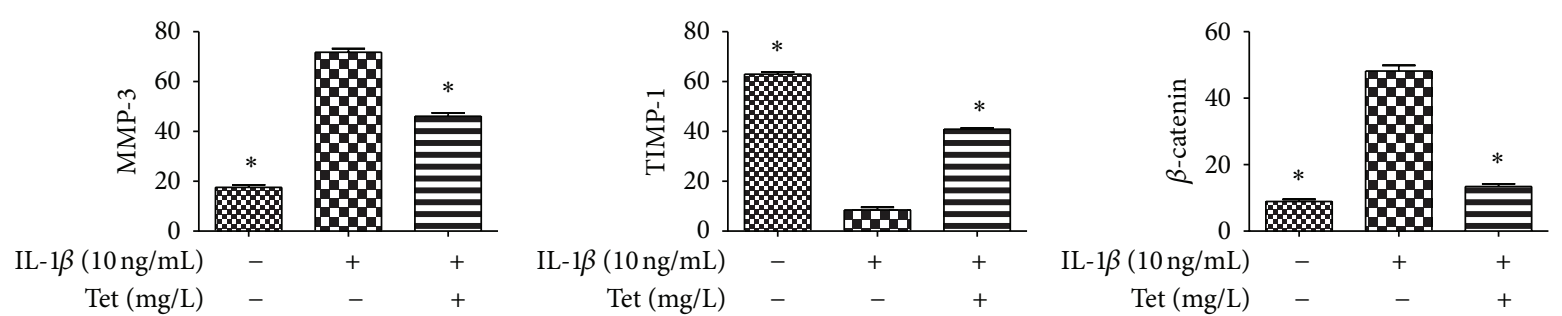

(b)

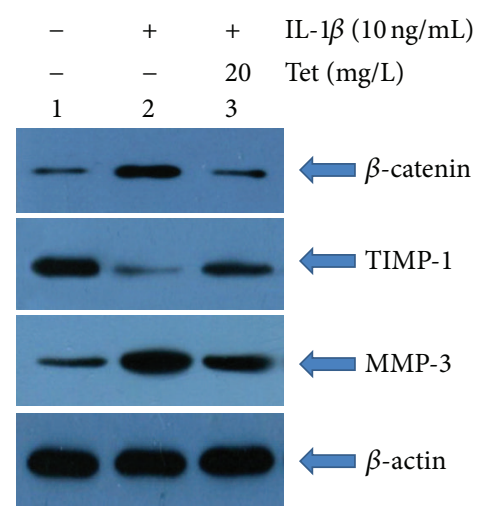

(c)

FIGURE 2: Effects of Tet on the expression of matrix metalloproteinase-1 (MMP-1), MMP-3, MMP-13, tissue inhibitors of metalloproteinase1 (TIMP-1), and $\beta$-catenin in chondrocytes. Cells were pretreated with Tet $(0 \mathrm{mg} / \mathrm{L}, 5 \mathrm{mg} / \mathrm{L}, 10 \mathrm{mg} / \mathrm{L}, 20 \mathrm{mg} / \mathrm{L})$ for one hour, followed by stimulation with interleukin-1 $\beta$ (IL-1 $\beta)(10 \mathrm{ng} / \mathrm{mL})$ for $24 \mathrm{~h}$. qRT-PCR was performed to determine the expression levels of MMPs in chondrocytes in (a). The protein concentrations of MMP-3, TIMP-1, and $\beta$-catenin were detected by Western blot analysis with the optimal concentration (b). The protein concentrations of MMP-3, MMP-13, and TIMP-1 were quantitated (c). Data are expressed as mean \pm SD. ${ }^{*} P<0.05$ compared with cells stimulated with IL-1 $\beta$ alone.

3.3. Macroscopic Observations. In the sham-operation (control) group, the cartilage on the femoral condyles was macroscopically normal, with a smooth, glistening surface, and no cartilage defect or osteophyte was observed. In the OA group, general characteristics of $\mathrm{OA}$, including erosion and osteophyte formation, were seen on the side of the femoral condyles after surgery. The Tet group showed less bone wear than the OA group as determined by gross appearance (Figure 3(b)).
3.4. Histopathological Changes in Articular Cartilage. Histopathological changes in the rabbits after surgery centred mainly on the thinner cartilage layer, abraded surface, and reduced safranin $\mathrm{O}$-fast green staining in the cartilage. Tet inhibited the cartilage degradation, which developed as OA progressed. However, osteophyte proliferation could not be reversed (Figure 3(c)). Consistent with these findings, the Mankin score was reduced in the Tet group compared with the OA group (Table 2 and Figure 4). 

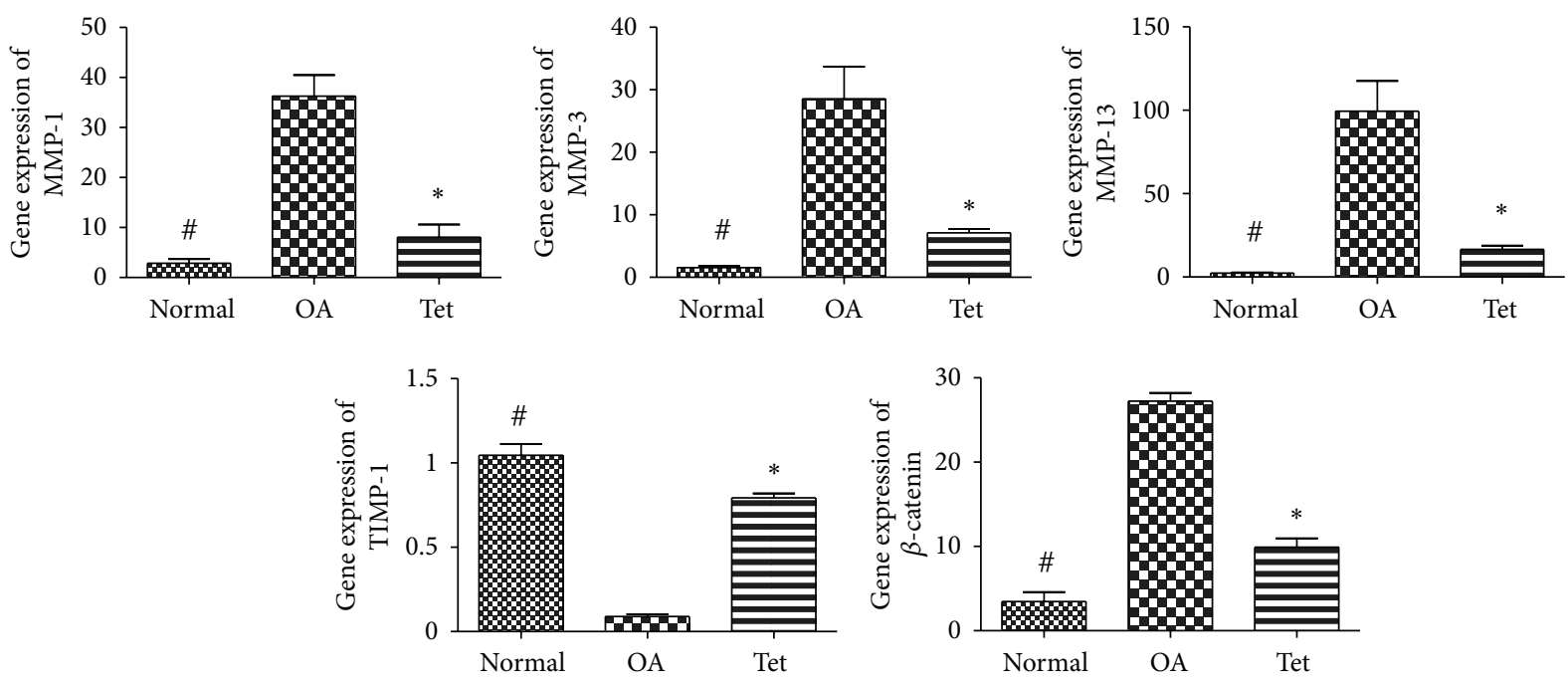

(a)
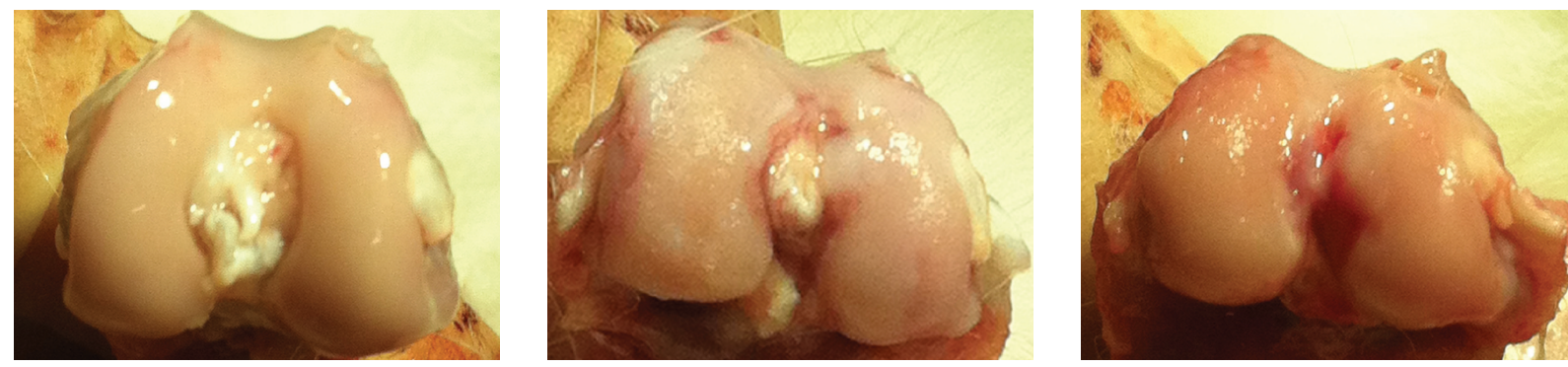

(b)

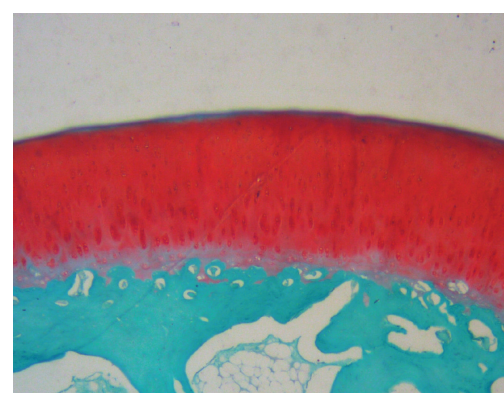

Normal

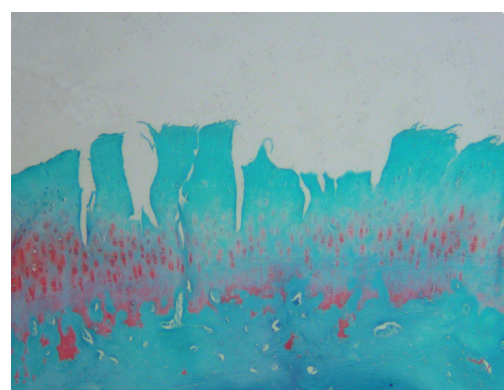

$\mathrm{OA}$

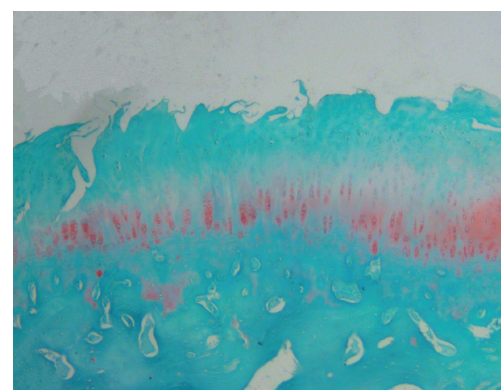

Tet

(c)

FIGURE 3: Effects of Tet on the expression of MMP-1, MMP-3, MMP-13, TIMP-1, and $\beta$-catenin in vivo experiment on cartilage in (a). The Tet and OA groups were treated with ACLT, and only the Tet groupwas given treatment of Tet $(20 \mathrm{mg} / \mathrm{L})$ for 6 weeks after surgery. Rabbits were sacrificed after last injection in articular cavity. Rabbits receive the sham-operation regarding as the Normal group. Representative pictures of macroscopic observations in (b) and Safranin O staining are shown in (c).

\section{Discussion}

OA may be of unknown origin (idiopathic, primary) or related to a known medical condition or event; the major pathological changes occur in the structure of the hyaline cartilage, with a variable degree of synovial inflammation. These changes are ascribed to a complex network of biochemical factors, including proteolytic enzymes, matrix metalloproteinases, and cytokines, which interact and lead to the breakdown of cartilage macromolecules. Cytokines, such as
TNF- $\alpha$ and IL-1 produced by mononuclear cells, activated synoviocytes, or even articular cartilage itself, significantly upregulate MMP gene expression [17]. As a result, cytokines affect compensatory chondrocyte synthesis pathways, leading to the degradation of the extracellular matrix (ECM). Many studies have demonstrated that IL-1 and TNF- $\alpha$ inhibit chondrocyte compensatory biosynthesis pathways, which can further compromise cartilage repair [18]. IL-1 $\beta$ is known to play a pivotal role in cartilage degradation, through the induction of MMPs secreted by chondrocytes. Chondrocytes 
TABLE 2: Histological score of articular cartilage.

\begin{tabular}{lccr}
\hline Femoral condyle & Normal group & Tet group & OA group \\
\hline Structural changes & $0.2 \pm 0.40$ & $3.0 \pm 0.63^{*}$ & $3.8 \pm 1.17^{\#}$ \\
Cellular changes & $0.4 \pm 0.49$ & $1.6 \pm 0.80^{*}$ & $1.8 \pm 0.75^{\#}$ \\
Safranin staining & $0.4 \pm 0.49$ & $1.8 \pm 0.75^{*}$ & $2.2 \pm 0.75^{\#}$ \\
Tide mark & $0.2 \pm 0.4$ & $0.4 \pm 0.49^{*}$ & $0.6 \pm 0.49^{\#}$ \\
Sum of score & $1.2 \pm 1.17$ & $6.8 \pm 1.33^{*}$ & $8.4 \pm 1.85^{\#}$ \\
\hline
\end{tabular}

Values are the means \pm SD. ${ }^{\#} P<0.05$ when Normal group compared with OA group, ${ }^{*} P<0.05$ when Normal group compared with Tet group.

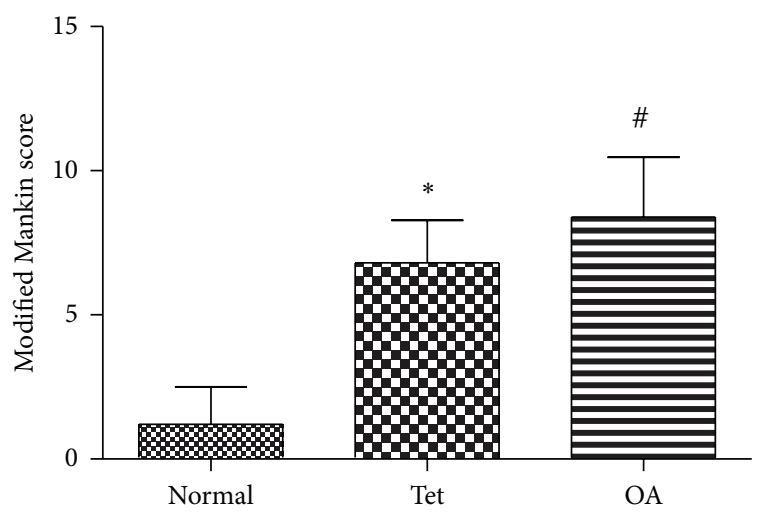

FIGURE 4: The Mankin scores are also presented. ${ }^{*} P<0.05$ when Normal group compared with OA group; ${ }^{*} P<0.05$ when Normal group compared with Tet group.

stimulated with IL-1 $\beta$ in vitro have been used to mimic the microenvironment that occurs in OA [19].

In this in vitro study, we used IL-1 $\beta$ to induce MMP gene expression, based on the hypothesis above, then evaluated the effect of Tet on MMP induction in rabbit chondrocytes, as well as its effects on ACLT-induced OA in the rabbit model [20]. We demonstrated that Tet inhibited the IL- $1 \beta$-mediated induction of MMP-1, MMP-3, and MMP-13 and induced the expression of TIMP-1, a metalloproteinase inhibitor, in rabbit articular chondrocytes; these effects were manifested at both the mRNA and protein levels. The MMPs are a family of proteinases that are involved in ECM degradation. MMP-1 and MMP-13, in particular, are interstitial collagenases that degrade type II collagen in cartilage, a major component of the ECM, which is a committed step in the progression of OA. As a result, targeting MMPs represents a promising approach to the treatment of OA. MMPs can be induced by IL-1 $\beta$, and increased expression of MMPs has been identified in osteoarthritic cartilage [21]. Similarly, serum concentrations of MMP-3 are elevated in patients with OA compared with normal subjects [22].

In the present study, pretreatment with $20 \mathrm{mg} / \mathrm{L}$ Tet resulted in significantly decreased expression levels of MMP1 , MMP-3, and MMP-13 in IL- $1 \beta$-induced chondrocytes. Tet also induced the expression of TIMP-1 without causing cytotoxicity. Because TIMPs are endogenous inhibitors of MMPs, we suggest that Tet may modulate the balance between
MMP and TIMP expression to exert its antiarthritic effects in IL-1 $\beta$-treated rabbit articular chondrocytes [23]. We also investigated in vivo the effects of Tet on cartilage degradation in an ACLT-based experimental model of OA in rabbits. This model has been widely used to evaluate the efficacy of agents in the treatment of OA due to the model's mechanical instability, which leads to cartilage degradation [24]. As shown in this study, ACLT in the rabbit resulted in cartilage degradation, and injection of Tet into the articular cavity $(0.3 \mathrm{~mL}, 20 \mathrm{mg} / \mathrm{L})$ for 6 weeks inhibited cartilage degradation as assessed by histological evaluation. The results were consistent with the in vitro findings. These observations provide evidence that Tet possesses chondroprotective activity both in vitro and in vivo.

Several signalling pathways are involved in the induction of MMPs by IL- $1 \beta$ in chondrocytes. Among them, the Wnt $/ \beta$ catenin signalling pathway and proteins regulate organ development, tumourigenesis, and bone homeostasis, among other functions, and are believed to play important roles in the induction of MMP expression [25, 26]. Given this, agents that interfere with the Wnt/ $\beta$-catenin signalling pathway may affect MMP expression. In the present study, we investigated whether Tet affected activation depending on the Wnt $/ \beta$ catenin signalling pathway. $\beta$-catenin is a multifunctional protein, involved in the formation of adhesive "tape" by interacting with cadherin at cell junctions. Furthermore, free $\beta$-catenin can penetrate into the nucleus to affect gene expression [27].

Our in vitro and in vivo findings suggest that expression of MMP and $\beta$-catenin genes showed a similar trend, as did the protein levels. We demonstrated that Tet effectively targets the Wnt $/ \beta$-catenin signalling pathway in chondrocytes, suggesting that inhibition of the Wnt/ $\beta$-catenin signalling pathway may, at least in part, account for changes in the trends in the expression of MMPs and TIMP-1, as well as cartilage protection. This study is, to our knowledge, the first to report the effects of Tet on Wnt/ $\beta$-catenin signalling in chondrocytes and in an ACLT-based experimental model of $\mathrm{OA}$ in rabbits. These findings are consistent with previous studies of the effects of Tet on the Wnt $/ \beta$-catenin signalling pathway in other cell systems [14]. The discrepancies between our findings and these reports may be related to differences in cell type and stimulation conditions.

In conclusion, modulation of cytokines, such as IL-1 $\beta$ and TNF- $\alpha$, which control MMP gene overexpression, appears to be a fertile target for drug development for the treatment of OA. Several studies have illustrated the potential importance 
of modulating IL-1 activity as a means to reduce the progression of structural changes in OA [24]. In the present study, we demonstrated that Tet possessed chondroprotective effects in IL- $1 \beta$-induced rabbit chondrocytes and an experimental model of OA. The inhibition of MMPs by Tet was associated at least in part with inhibition of the $\mathrm{Wnt} / \beta$-catenin signalling pathway. Our results indicate that Tet shows promise as a therapeutic agent for the treatment of OA. However, further studies are needed to confirm and extend these preliminary findings.

\section{Conflict of Interests}

The authors declare that they have no conflict of interests.

\section{Authors' Contribution}

X. Zhou and L. Wu designed the research; X. Zhou, W. Li, and L. Jiang performed the research; X. Zhou and J. Bao, L. Tao, and J. Li analyzed the data, and X. Zhou wrote the paper.

\section{Acknowledgment}

This study was supported by the National Natural Science Foundation of China (81071492).

\section{References}

[1] D. T. Felson, "Epidemiology of hip and knee osteoarthritis," Epidemiologic Reviews, vol. 10, pp. 1-28, 1988.

[2] M. B. Goldring, "The role of the chondrocyte in osteoarthritis," Arthritis \& Rheumatism, vol. 43, no. 9, pp. 1916-1926, 2000.

[3] M. Gowen, D. D. Wood, and R. G. G. Russell, "Stimulation of the proliferation of human bone cells in vitro by human monocyte products with interleukin-1 activity," Journal of Clinical Investigation, vol. 75, no. 4, pp. 1223-1229, 1985.

[4] J. A. Mengshol, M. P. Vincenti, C. I. Coon, A. Barchowsky, and C. E. Brinckerhoff, "Interleukin-1 induction of collagenase 3 (matrix metalloproteinase 13) gene expression in chondrocytes requires p38, c-Jun $\mathrm{N}$-terminal kinase, and nuclear factor kappaB: differential regulation of collagenase 1 and collagenase 3," Arthritis \& Rheumatism, vol. 43, no. 4, pp. 801-811, 2000.

[5] N. Ishiguro, T. Ito, H. Ito et al., "Relationship of matrix metalloproteinases and their inhibitors to cartilage proteoglycan and collagen turnover: analyses of synovial fluid from patients with osteoarthritis," Arthritis \& Rheumatism, vol. 42, no. 1, pp. 129136, 2001.

[6] Y. Okada, M. Shinmei, O. Tanaka et al., "Localization of matrix metalloproteinase 3 (stromelysin) in osteoarthritic cartilage and synovium," Laboratory Investigation, vol. 66, no. 6, pp. 680-690, 1992.

[7] R. M. Hembry, M. R. Bagga, J. J. Reynolds, and D. L. Hamblen, "Immunolocalisation studies on six matrix metalloproteinases and their inhibitors, TIMP-1 and TIMP-2, in synovia from patients with osteo- and rheumatoid arthritis," Annals of the Rheumatic Diseases, vol. 54, no. 1, pp. 25-32, 1995.

[8] I. Uthman, J. P. Raynauld, and B. Haraoui, "Intra-articular therapy in osteoarthritis," Postgraduate Medical Journal, vol. 79, no. 934, pp. 449-453, 2003.
[9] N. H. Mashour, G. I. Lin, and W. H. Frishman, "Herbal medicine for the treatment of cardiovascular disease: clinical considerations," Archives of Internal Medicine, vol. 158, no. 20, pp. 2225-2234, 1998.

[10] X. F. Yu, C. Q. Zou, and M. B. Lin, "Observation of the effect of tetrandrine on experimental silicosis of rats," Ecotoxicology and Environmental Safety, vol. 7, no. 3, pp. 306-312, 1983.

[11] L. J. Ho and J. H. Lai, "Chinese herbs as immunomodulators and potential disease-modifying antirheumatic drugs in autoimmune disorders," Current Drug Metabolism, vol. 5, no. 2, pp. 181192, 2004.

[12] H. S. Kim, Y. H. Zhang, and Y. P. Yun, "Effects of tetrandrine and fangchinoline on experimental thrombosis in mice and human platelet aggregation," Planta Medica, vol. 65, no. 2, pp. 135-138, 1999.

[13] F. P. Luyten, P. Tylzanowski, and R. J. Lories, "Wnt signaling and osteoarthritis," Bone, vol. 44, no. 4, pp. 522-527, 2009.

[14] B. C. He, J. L. Gao, B. Q. Zhang et al., "Tetrandrine inhibits Wnt/beta-catenin signaling and suppresses tumor growth of human colorectal cancer," Molecular Pharmacology, vol. 79, no. 2, pp. 211-219, 2011.

[15] L. J. Brunet, J. A. McMahon, A. P. McMahon, and R. M. Harland, "Noggin, cartilage morphogenesis, and joint formation in the mammalian skeleton," Science, vol. 280, no. 5368, pp. 1455-1457, 1998.

[16] H. J. Mankin, M. E. Johnson, and L. Lippiello, "Biochemical and metabolic abnormalities in articular cartilage from osteoarthritic human hips. III. Distribution and metabolism of amino sugar-containing macromolecules," Journal of Bone and Joint Surgery A, vol. 63, no. 1, pp. 131-139, 1981.

[17] J. C. Fernandes, J. Martel-Pelletier, and J. P. Pelletier, "The role of cytokines in osteoarthritis pathophysiology," Biorheology, vol. 39, no. 1-2, pp. 237-246, 2002.

[18] M. Lotz, "Cytokines in cartilage injury and repair," Clinical Orthopaedics and Related Research, supplement 391, pp. S108S115, 2001.

[19] F. Moldovan, J. P. Pelletier, F. C. Jolicoeur, J. M. Cloutier, and J. Martel-Pelletier, "Diacerhein and rhein reduce the ICE-induced IL-1 $\beta$ and IL-18 activation in human osteoarthritic cartilage," Osteoarthritis and Cartilage, vol. 8, no. 3, pp. 186-196, 2000.

[20] P. F. Hu, Y. Chen, P. F. Cai, L. F. Jiang, and L. D. Wu, "Sphingosine-1-phosphate: a potential therapeutic target for rheumatoid arthritis," Molecular Biology Reports, vol. 38, no. 6, pp. 4225-4230, 2011.

[21] M. P. Vincenti and C. E. Brinckerhoff, "Transcriptional regulation of collagenase (MMP-1, MMP-13) genes in arthritis: integration of complex signaling pathways for the recruitment of gene-specific transcription factors," Arthritis Research, vol. 4, no. 3, pp. 157-164, 2002.

[22] L. S. Lohmander, P. J. Neame, and J. D. Sandy, "The structure of aggrecan fragments in human synovial fluid: evidence that aggrecanase mediates cartilage degradation in inflammatory joint disease, joint injury, and osteoarthritis," Arthritis and Rheumatism, vol. 36, no. 9, pp. 1214-1222, 1993.

[23] D. D. Dean, J. Martel-Pelletier, J. P. Pelletier, D. S. Howell, and J. F. Woessner, "Evidence for metalloproteinase inhibitor imbalance in human osteoarthritic cartilage," Journal of Clinical Investigation, vol. 84, no. 2, pp. 678-685, 1989.

[24] W. P. Chen, P. F. Hu, J. P. Bao, and L. D. Wu, "Morin exerts antiosteoarthritic properties: an in vitro and in vivo study," Experimental Biology and Medicine, vol. 237, no. 4, pp. 380-386, 2012. 
[25] G. Schett, J. Zwerina, and J. P. David, “The role of Wnt proteins in arthritis," Nature Clinical Practice Rheumatology, vol. 4, no. 9, pp. 473-480, 2008.

[26] T. Yuasa, T. Otani, T. Koike, M. Iwamoto, and M. EnomotoIwamoto, "Wnt/ $\beta$-catenin signaling stimulates matrix catabolic genes and activity in articular chondrocytes: is possible role in joint degeneration," Laboratory Investigation, vol. 88, no. 3, pp. 264-274, 2008.

[27] M. Corr, "Wnt- $\beta$-catenin signaling in the pathogenesis of osteoarthritis," Nature Clinical Practice Rheumatology, vol. 4, no. 10, pp. 550-556, 2008. 


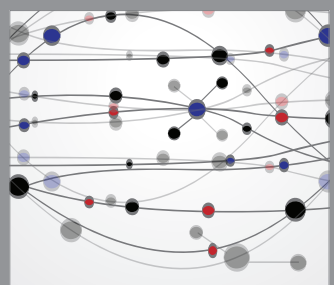

The Scientific World Journal
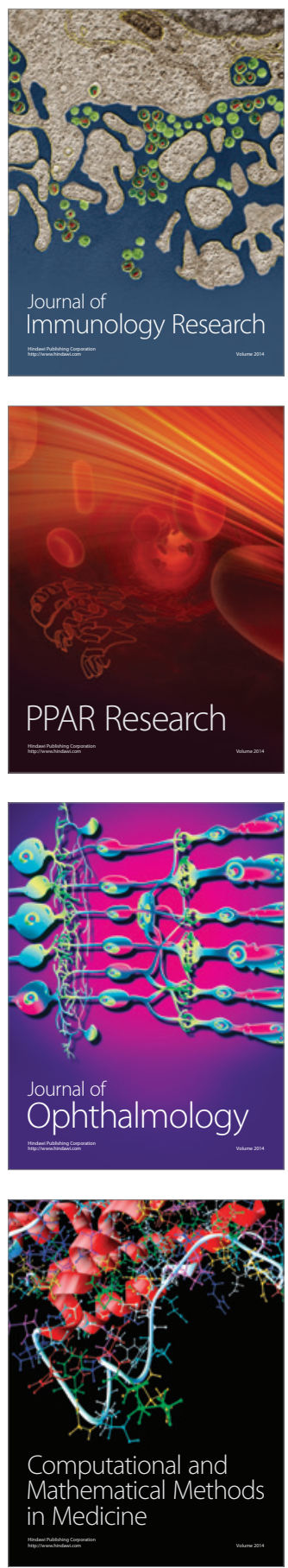

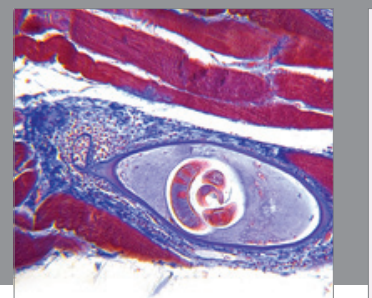

Gastroenterology

Research and Practice
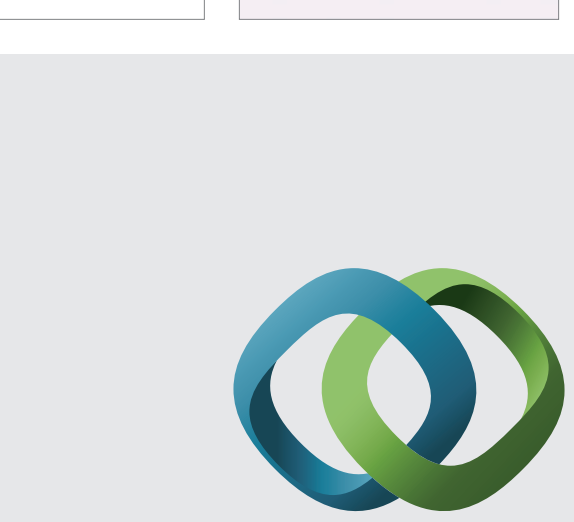

\section{Hindawi}

Submit your manuscripts at

http://www.hindawi.com
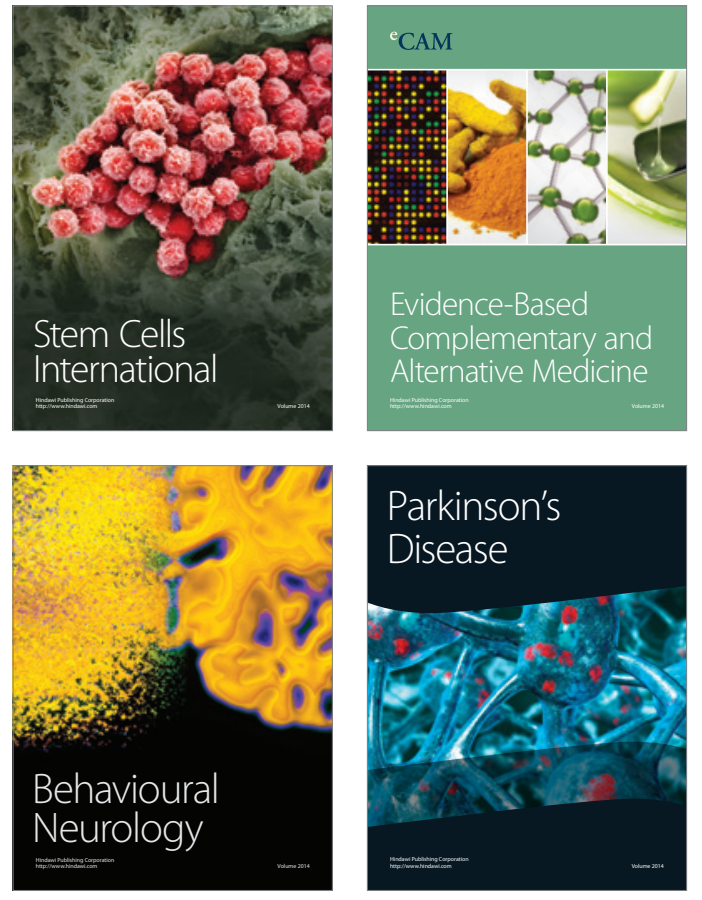
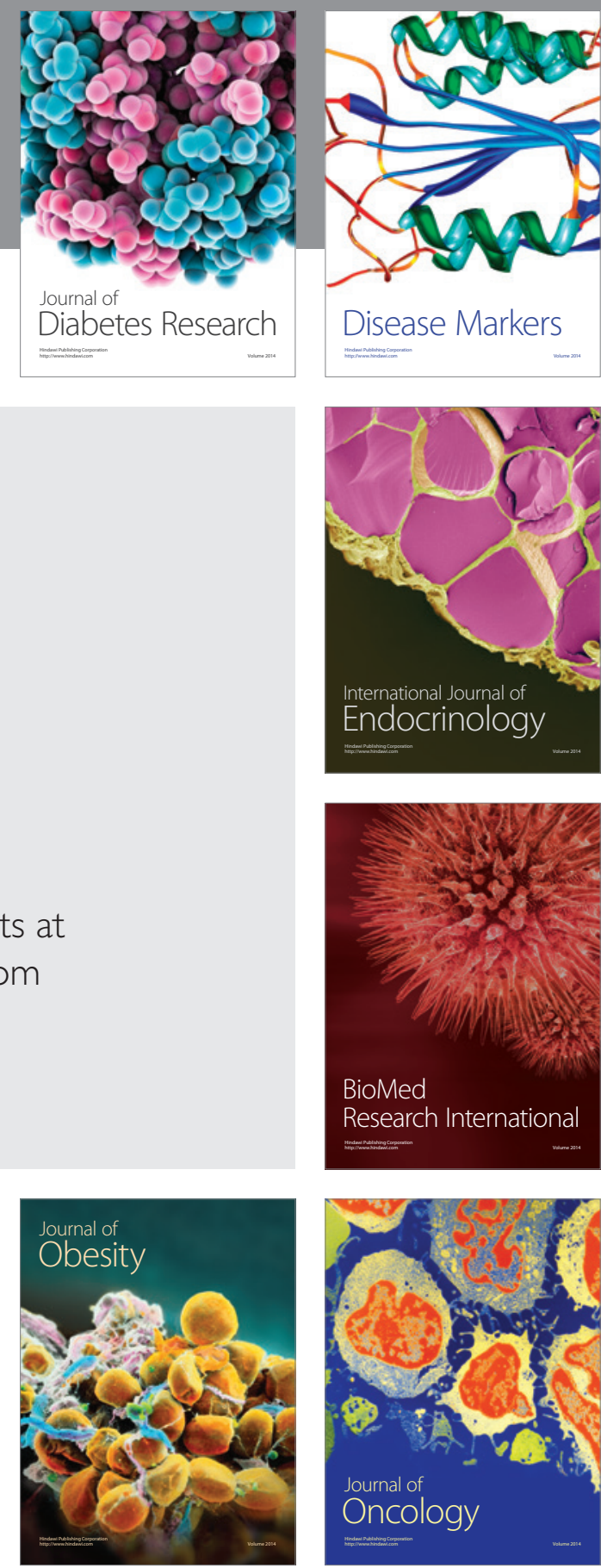

Disease Markers
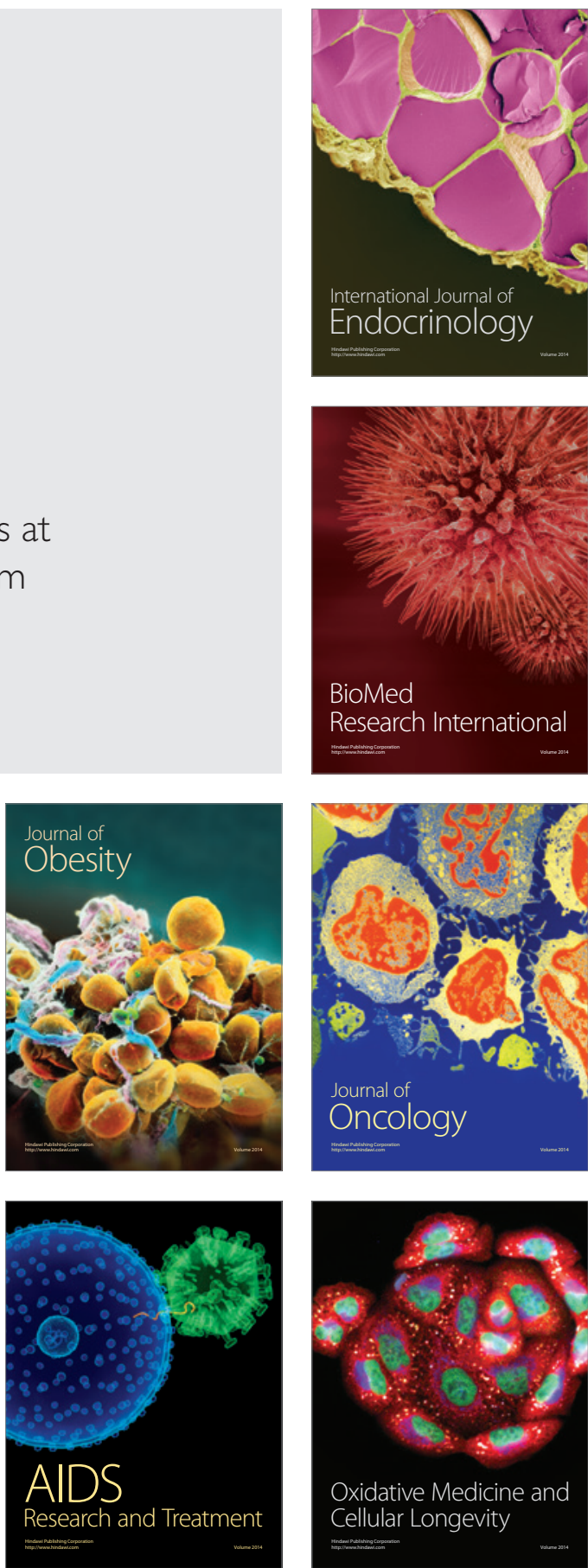